\title{
Richtwirkung verschiedener lonen auf anisotrope Kolloide
}

\author{
Von HeinRich Thiele und Hans Lück \\ Aus der Chemischen Abteilung am Hygienischen Institut der Universität Köln \\ (Z. Naturforschg. 3 b, 393-402 [1948]; eingegangen 2. Oktober 1948)
}

\begin{abstract}
Anisodiametrische Teilchen von Solen können durch oberflächenaktive Stoffe, durch Phasengrenzen oder durch Elektrolyte ausgerichtet werden.

Gemessene und berechnete Diffusionspotentiale der ionogenen Stoffe ergeben eine Beziehung zwischen der Aufladung des Gelrandes, bedingt durch die verschiedene Diffusionsgeschwindigkeit der Ionen, und dem Vorzeichen der Doppelbrechung.

Durch die positive oder negative Aufladung des Gelrandes werden die Teilchen im Sol durch Anziehung oder Abstoßung radial oder tangential ausgerichtet - und in dieser Lage fortschreitend fixiert.
\end{abstract}

$\mathrm{K}$ olloide Lösungen mit Teilchen von laminarer oder fibrillarer Gestalt werden unter bestimmteñ Bedingungen doppelbrechend. Man unterscheidet Form- und Eigendoppelbrechung. Die Formdoppelbrechung ${ }^{1}$ entsteht durch gleichmäßige Anordnung von anisodiametrischen, isotropen Teilchen dann, wenn ihre Brechungsindices von dem des zwischen ihnen befindlichen Mediums verschieden sind. Eigendoppelbrechung setzt das Vorhandensein anisotroper Teilchen voraus. Bei den folgenden Betrachtungen handelt es sich um eigendoppelbrechende Sole.

Die Kräfte zur Ausrichtung der Solteilchen können verschiedener Art sein. Stäbchen und Plättchen einer kolloiden Lösung lassen sich durch elektrische ${ }^{2}$ und magnetische ${ }^{3}$ Felder sowie mechanisch durch Strömung ${ }^{4}$ ausrichten. Wie sich zeigte, sind auch Elektrolyte und oberflächenaktive Stoffe hierzu imstande ${ }^{5}$.

Bringt man einen Salzkristall in ein Sol mit anisotropen Teilchen, so entsteht um den Kristall herum durch Flockung allmählich ein Gel. Neuer Elektrolyt diffundiert nach und läßt das Gel schließlich bis an die Wand des Gefäßes anwachsen. In vielen Fällen geschieht dieser Vorgang als eine gerichtete Koagulation. Diese gerichtete Koagulation haben wir mit Ionotropie bezeichnet, nach ionos $=$ Ion und tropos $=$ Gestalt ${ }^{6}$. Führt man den Versuch in einer flachen Glasschale aus und betrachtet die kreisrunde Gel-

1 Ber. Verh. sächs. Ges. Akad. Wiss. Leipzig, math.physische Kl. 61, 113 [1929].

2 Diesselhorst, Freundich u. Leonh a r d t, Elster- u. Geitel-Festschrift, Braunschweig 1915 , S. 453.

${ }^{3}$ M a jo r a n a, Atti R. Accad. naz. Lincei, Rend. 11 I, $374,463,531$ [1902]. scheibe unter dem Polariskop, so sieht man das Bild eines doppelbrechenden, einachsigen Kristalls.

Hierzu kann man ein Vanadinpentoxyd-Sol verwenden. Dieses Sol enthält neben molekular gelöstem Anteil viele feine doppelbrechende Nadeln

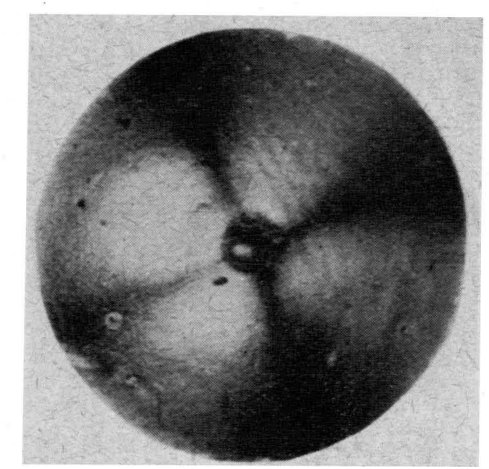

lbb. 1. Ionotropes Gel, erhalten aus $\mathrm{V}_{2} \mathrm{O}_{5}$-'Sol mit $\mathrm{NaNO}_{3}$ im polarisierten Licht bei gekreuzten Nicols. Vergrößerung $3 / 1$.

verschiedener Größe. Nach Röntgenaufnahmen besteht das Gitter des Vanadinpentoxyds aus einem ebenen Netz von $\mathrm{VO}_{4}$-Tetraedern. Diese fibrillare Gestalt des Kriställchens hat ihre Ursache in der leichten Spaltbarkeit in Richtung der in Abb. 3 schraffiert gezeichneten Tetraeder. Die längere Achse des Indexellipsoides liegt in der Längsrichtung der Nadeln, die kürzere senkrecht dazu. Um dies festzustellen, bringt man im Polarisationsmikroskop über das Objekt ein Gipsplättchen Rot I. Ordnung. Fällt die längere Index-

4 Diesselhorst, Freundlich u. Leonha rdt, Physik. Z. 16, 413 [1915].

5 H. Th i e l e, Naturwiss. 34, 123 [1947].

"H. Th i e l e, Z. Naturforschg. 3 b, 7 [1948]. 
achse von Gips und $\mathrm{V}_{2} \mathrm{O}_{5}$ - Kristall zusammen, so tritt Addition des Gangunterschiedes von Gips und Objekt, damit Farbvertiefung auf. Stehen sie senkrecht zueinander, so beobachtet man durch Subtraktion des Gangunterschiedes Farbaufhellung. Im ersten Fall erscheint das Kriställchen bei $\mathrm{V}_{2} \mathrm{O}_{5}$ grün-schwarz gezeichnet, im zweiten Fall ist es gelborange (Abb.2). läßt, wird vom Kristall umgewandelt in die beiden Richtungen der Indexachsen. Beide Strahlen erhalten im Objekt einen Gangunterschied. Ihre Vereinigung beim Verlassen des Kristalls führt $\mathrm{zu}$ einer Schwingung, die nicht mehr senkrecht zum Analysator steht. Daher herrscht in diesen Richtungen Helligkeit. Das dunkle Kreuz liegt parallel zum Polarisator und Analysator (Abb.4).

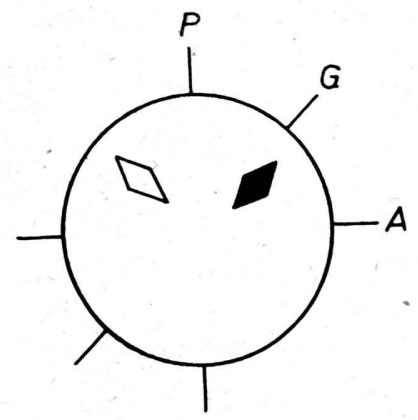

Abb. 2.

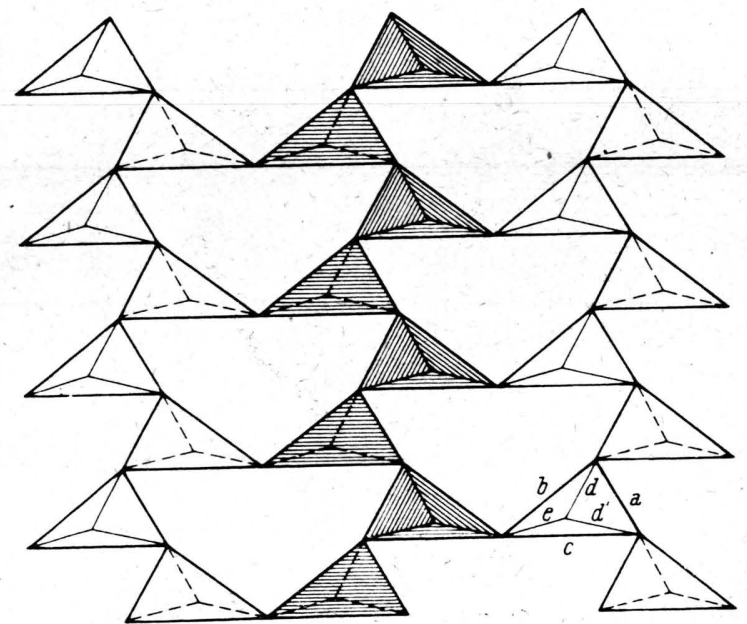

Abb. 1 kommt dadurch zustande, daß sich die Solteilchen erst ausrichten und dann ein Gel bilden. Dabei liegen drei Möglichkeiten vor. Die Teilchen können sich ungeordnet festlegen, oder sie können sich radial oder tangential ordnen. In den beiden letzteren Fällen findet man ein Achsenkreuz. Teilchen, welche in Richtung des Polarisators oder Analysators liegen, vérändern die Lichtschwingung nicht. Deshalb bleibt Dunkelheit, wie es auch ohne Objekt der Fall ist. In allen anderen Lagen tritt eine Drehung der Lichtschwingung durch das Teilchen auf. Die Schwingungsrichtung des Lichtes, die der Polarisator durch-
Abb. 2. Verschiedenes Verhalten von $\mathrm{V}_{2} \mathrm{O}_{5}$ - Kristallen nach ihrer Lage zum Gips. Richtung der Lichtschwingung im Analysator $=\mathrm{A}$, im Polarisator $=\mathrm{P}$, $\mathrm{G}=$ längere Indexachse des Gipses.

Abb. 3. Ebenes Netz aus verzerrten $\mathrm{VO}_{4}$-Tetraedern im Vanadinpentoxyd. Die Schnittpunkte der dünn aus ${ }^{2}$ .gezogenen Tetraederkanten sind oberhalb, die der gestrichelten unterhalb der Zeichenebene liegend $\mathrm{zu}$ denken. Die Tetraederkantenlängen betragen $\mathrm{a}=2,13$, $\mathrm{b}=3,06, \mathrm{c}=3,50, \mathrm{~d}=\mathrm{d}^{\prime}=2,72, \mathrm{e}=2,36$; der mittlere Abstand V-0 beträgt 1,72 ̊ (nach Remy, ,Anorganische Chemie“).

Abb. 4. Radiale und tangentiale Anordnung der Kolloidteilchen in ionotropen Gelen.

Abb. 3.

Bei der Strömungsdoppelbrechung nach der Wirbelmethode erscheint dasselbe Bild, nur daß hierbei das Kreuz um einen Winkel $\varphi$ verdreht ist. Z o cher führt dies auf eine Scherung der Teilchen im Wirbelfeld zurück ${ }^{7}$.

Bei ionotropen Gelen tritt keine Scherung auf. Daher ist hier $\varphi=0^{\circ}$ oder $90^{\circ}$.

Bei der Untersuchung einer Reihe von Salzen mit $\mathrm{V}_{2} \mathrm{O}_{5}$-Sol ergaben sich isotrope wie auch positiv und negativ doppelbrechende Gele. In Analogie $\mathrm{zu}$ Strömungsversuchen mit $\mathrm{V}_{2} \mathrm{O}_{5}$-Sol nach der Wirbelmethode definieren wir ein Gel als positiv

' H. Z o c h e r, Z. physik. Chem. 114, 193 [1924]. 
doppelbrechend, wenn die farbigen Sektoren, die eine Farbvertiefung ergeben, senkrecht zur Richtung der Schwingung des Gipses stehen, und als negativ doppelbrechend, wenn sie parallel dazu liegen.

Um festzustellen, welche Elektrolyte positiv und welche negativ richtend wirken, war es erforderlich, ausgedehnte Reihenversuche mit Salzen aus allen Gruppen des periodischen Systems anzustellen.

Vanadinpentoxydsol wird nach Biltz durch Verreiben von $\mathrm{NH}_{4} \mathrm{VO}_{3}$ in verd. $\mathrm{HCl}$ in der Achatschale erhalten, indem man danach filtriert und mit Wasser so lange nachwäscht, bis die anfangs klare Lösung kolloid durchzulaufen beginnt. Den Filterrüiekstand peptisiert man unter Schütteln mit Wasser zu dem klaren tiefroten Sol, welches nach einigen Tagen Strömungsdoppelbrechung zeigt.

Gleichzeitig wurde auch der Einfluß auf ein über 10 Jahre altes Eisenhydroxydsol aus Liquor ferri oxychlorati dialysati untersucht. Das Sol zeigte starke Strömungsdoppelbrechung. Unter dem Polarisationsmikroskop kann man Stäbchen oder längliche Scheibchen sehen, die in der Richtung des Gipses grün, senkrecht dazu gelb bis braun erscheinen. Hergestellt war das Sol nach dem Hydrolysenverfahren. Man neutralisiert $\mathrm{FeCl}_{3}$ nicht vollständig mit $\mathrm{NH}_{4} \mathrm{OH}$ und dialysiert anschließend. Frische Sole, die keine Strömungsdoppelbrechung zeigten, lieferten isotrope Gelscheiben. Versuche, solche durch Wechselstrom künstlich $\mathrm{zu}$ altern, wie es in der Literatur angegeben ist, blieben erfolglos.

Betrachtet man die Ergebnisse der Versuchsreihen mit rd. 150 Salzen aus allen Gruppen des periodischen Systems, so läßt sich ohne weiteres keine Ordnung erkennen. Die Wertigkeit der richtenden Ionen allein ist nicht bestimmend. Aber es fällt auf, daß die Doppelbrechung in $\mathrm{V}_{2} \mathrm{O}_{5}$ und $\mathrm{Fe}(\mathrm{OH})_{3}$ bei vielen Salzen für dasselbe Ion entgegengesetzt ist. Wie wir später sehen werden, ist die Äquivalentleitfähigkeit und damit die Wanderungsgeschwindigkeit der beiden Ionen von Einfluß.

Voraussetzung für die Deutungsversuche der verschiedenartigen Teilcheneinstellung war die Tatsache, daß die Teilchen selbst ihre optischen Eigenschaften bei der Ionotropie nicht verändern.

Bei gleichbleibender Fixierung der Stäbchen im Gel könnte ein Effekt durch eine Änderung ihrer Eigendoppelbrechung eintreten, welche verursacht wäre durch eine Strukturänderung, eine Aneinanderlagerung oder Umlagerung der Teilchen.

Diese Vorgänge könnten eine Umkehrung der Achsen des Indexellipsoides zur Folge haben und damit eine Erklärung für die gefundene verschiedene Doppelbrechung geben, wie man sie gelegentlich in Seifensolen beobachtet. Diese Verschiedenartigkeit müßte sich dann auch im Sol näch der Wirbelmethode nachweisen lassen, wenn das Sol mit einer Elektrolytmenge versetzt wird, deren Konzentration bis fast an die Flockungsgrenze heranreicht. In zahlreichen Versuchen wurde dies aber nicht beobachtet. Bei $\mathrm{V}_{2} \mathrm{O}_{5}$ - und $\mathrm{Fe}(\mathrm{OH})_{3}$-Sol tritt keine Änderung der Strömungsdoppelbrechung vor der Koagulation durch das entsprechende Salz ein. Während oder nach der Flockung kann ebenfalls keine Umkehr der Indexachsen der Teilchen eintreten. Die Flockung ist ein kontinuierlicher Vorgang. Außerdem muß die Richtwirkung in jedem Falle der Koagulation vorausgehen.

Wie sich zeigen läßt, verändern die Teilchen ihre Lage nicht mehr, sofern nicht sekundär eine chemische Reaktion des Sols mit dem Salz einsetzt, die die Gelstruktur zerstört. Der größte Teil der Salze verändert die Teilchen selbst nicht.

Versuche, den Vorgang des Ausrichtens selbst bei der verschiedenartigen Einstellung der SoIteilchen unter dem Polarisationsmikroskop oder Ultramikroskop zu erbringen, sind bis jetzt nicht einwandfrei gelungen. Die größeren Teilchen folgen der Richtwirkung nicht deutlich, weil durch die starke Lichtquelle die Erwärmung im mikroskopischen Objekt störende Strömungen des Sols erzeugt. Die kleineren Teilchen konnten unter den gegebenen Bedingungen nicht mehr sichtbar gemacht werden.

Dagegen zeigen mikroskopische Bilder von fertig gebildeten ionotropen Gelen eindeutig, daß sich die Solteilchen einmal radial und einmal tangential eingestellt haben. So entsteht das verschiedene Vorzeichen der Doppelbrechung in diesen Gelen.

In den Reihenversuchen mit Salzen ist nicht ohne weiteres eine Gesetzmäßigkeit für die verschiedene Ausrichtung zu erkennen. Es liegt nahe, zur Erklärung der Erscheinungen zunächst eine Änderung der Oberflächenspannung durch Ionen anzunehmen. Oberflächenaktive Stoffe wie Campher, Thymol und Heptylalkohol erzeugen starke Doppelbrechung, ohne jedoch ein Gel zu bilden ${ }^{6}$. Das Kreuz verschwindet daher nach einiger Zeit durch die Wärmebewegung wieder. Gesättigte Salzlösungen weichen nur wenig von dem Wert für die Oberflächenspannung des Wassers ab, wie aus Tab. 1 zu ersehen ist. 


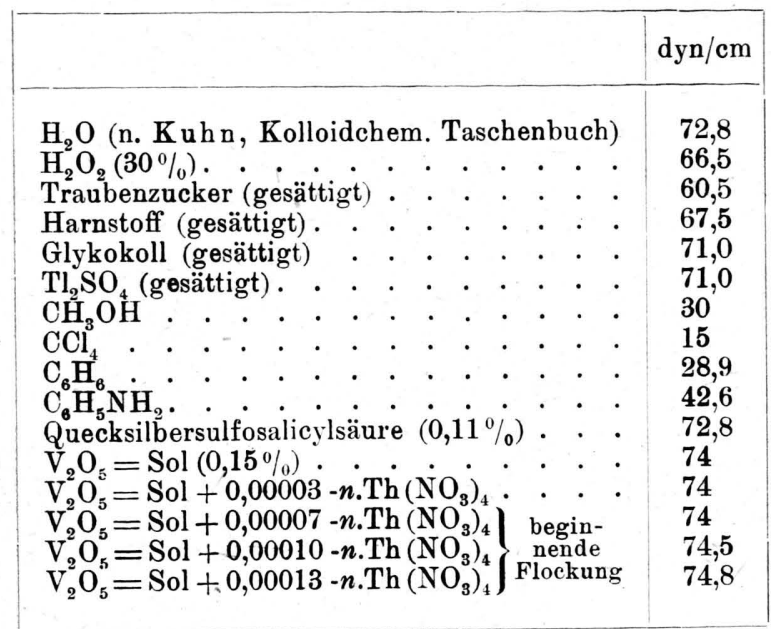

Tab. 1. Oberflächenspannung gegen Luft bei Zimmertemperatur (gemessen mit einem Stalagmometer).

Außerdem genügen häufig schon Millimole der Elektrolytlösungen, um aus dem Sol ein Gel zu bilden. Diese geringen Zusätze ändern die Oberflächenkräfte nicht nachweisbar, wie an einer Reihe von Lösungen und mit Elektrolyt versetzten Solen mit dem Stalagmometer nachgemessen wurde. Dasselbe gilt für bereits geflockte Sole. Daher kann die Änderung der Oberflächenspannung nicht für die verschiedenen Erscheinungen verantwortlich gemacht werden.

Bei unseren Versuchen ist die Oberflächenspannung gegen das betreffende Sol von Bedeutung. Da diese gegen wäßrige Lösungen nicht zu messen ist, kann die Tab. 1 nur qualitativ Auskunft geben, inwieweit Änderungen zu erwarten sind. Versuche, die Oberflächenspannung durch Messung der Steighöhe in Kapillaren zu bestimmen, konnten innerhalb der Fehlergrenze keinen Unterschied zwischen Sol und Grenzschicht Sol/Gel erkennen lassen.

Die Richtung der meisten nichtionogenen Substanzen wird allerdings auf ihre Oberflächenaktivität zurückzuführen sein.

Nach der Zusammenstellung in Tab. 2 stellen alle Nichtelektrolyte die Teilchen radial ein. Dabei ist die Richtwirkung nicht durch die entstandene Strömung des einfallenden Tropfens bedingt. Es genügt beim $\mathrm{V}_{2} \mathrm{O}_{5}$-Sol schon, einen Tropfen Methylalkohol oder einen Kristall Campher über die Oberfläche des Sols zu halten. um denselben Effekt wahrzunehmen. Die anderen Sole verhalten sich etwas träger. Doch bei einigen Flüssigkeiten wird auch hier eine Richtwir-

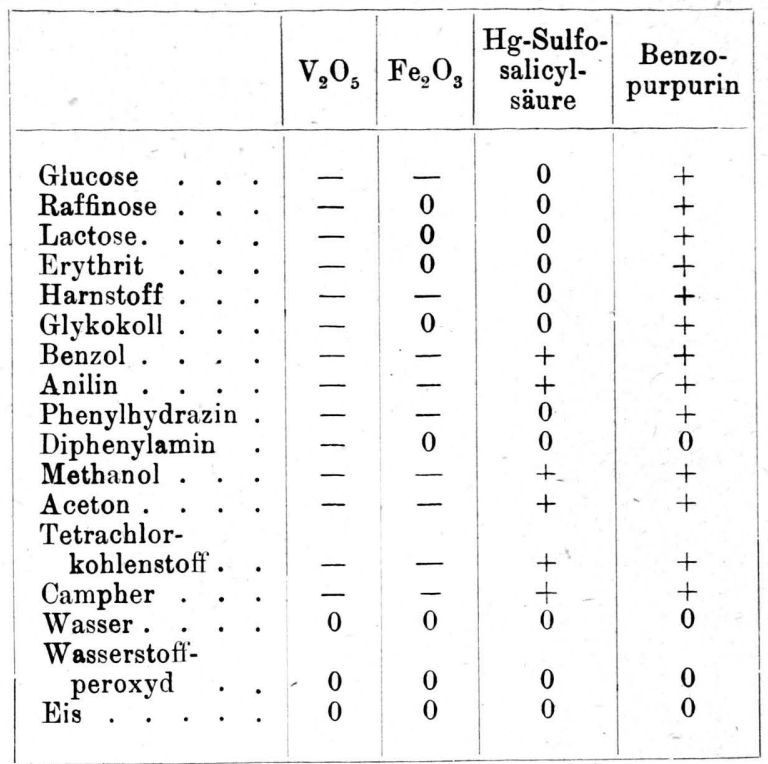

Tab. 2. Einfluß von Nichtelektrolyten auf die Doppelbrechung.

kung gefunden, ohne daß die Substanz das Sol berührt, lediglich durch die Einwirkung der Dämpfe. Bei Hg-Sulfosalicylsäure gelingt es, durch Hinzufügen von etwas Benzol und Aceton die Oberflächenspannung des Sols so weit zu erniedrigen, daß Campher nur noch eine schwache Doppelbrechung erzeugt. Daraus läßt sich erkennen, daß Oberflächenkräfte von Einfluß auf die Ausrichtung sind. Bei den festen Stoffen, wie den Zukkern, Harnstoff, bewirken die Moleküle des sich lösenden Stoffes die Orientierung. Dies findet in dem Maße statt, wie Zucker oder Harnstoff in die Lösung hineindiffundieren.

Allerdings entsteht hier die Schwierigkeit, ihre schwache Richtwirkung zu deuten. Diese Stoffe sollen nach Freundlich sogar kapillarinaktiv ${ }^{8}$ sein. Sie würden danach sogar die Oberflächenspannung erhöhen. Versuche mit gesättigten Lösungen dieser Stoffe zeigten eine schwache Erniedrigung. Man wird diese geringen Änderungen der Kräfte für die Ausrichtung kaum verantwortlich machen können. Wahrscheinlich liegen hier im Prinzip dieselben Ursachen wie bei der Strömungsdoppelbrechung vor.

Durch Diffusion der sich lösenden Substanz entsteht im Sol eine schwache Strömung. Ihre Geschwindigkeit wird keinesfalls in allen Richtungen die gleiche sein. In der Hydrodynamik besteht

s H. Freundlich, Kapillarchemie, Bd. I, S. 68. 
die bekannte Tatsache, daß sich Teilchen in einem Strömungsfeld mit ihrer Längsachse in die Richtung der Strömung einstellen, wenn ein Geschwindigkeitsgefälle senkrecht dazu besteht. Dies kann auch bei der Erklärung der Ausrichtung durch oberflächenaktive Stoffe herangezogen werden.

Je höher die Erniedrigung der Oberflächenspannung durch einen Stoff ist, um so schneller wird sich dieser in dem. Sol oder auf der Oberfläche ausbreiten. Die damit entstehende Strömung kann die Solteilchen ausrichten. Schließlich ist noch versucht worden, mit einer Kräfteänderung durch einseitige Aufladung der Grenzfläche des Gels die verschiedene Auswirkung zu erklären. Diese kann durch verschieden schnelle Wanderung von Kation und Anion bedingt sein. Jede Aufladung, gleichgültig ob positiv oder negativ, setzt durch gegenseitige Abstoßung der elektrisch geladenen Teilchen die Oberflächenspannung herab und würde nur die Orientierung in einer Richtung erklären können.

Für sie gilt die Gleichung:

$$
\sigma=\sigma_{0}-k V^{2} \text {, darin ist }
$$

$\sigma=$ Oberflächenspannung durch Aufladung,

$\sigma_{0}=$ Oberflächenspannung vor der Aufladung,

$k=$ eine Konstante,

$V=$ Potential der Aufladung.

Phasengrenzen können ebenfalls ausrichtend wirken. Bringt man eine kleine Glaskugel in ein Sol, so wird es an der Grenzfläche doppelbre. chend, wie Tab. 3 zeigt.

Graphitsäure ist ein Blättchensol. Die verwendete Seifenlösung besitzt Fibrillarstruktur und zeigte positive Strömungsdoppelbrechung. Quecksilbersulfosalicylsäure und Benzopurpurin sind ebenfalls Stäbchensole. Die längere Indexachse liegt im Gegensatz zu $\mathrm{V}_{2} \mathrm{O}_{5}$ und Fe-Oxydsol senkrecht zur Längsrichtung der Teilchen. Bei Eisenhydroxydsol waren an der Phasengrenze keine klaren Erscheinungen zu erkennen.

Die Teilchen lagern sich parallel zur Phasengrenze. Wenn dieser Effekt auch schwach ist, so muß man ihn doch berücksichtigen, ehe man eine Erklärung bei anderen Einflüssen sucht. Sieht man von einem einfachen mechanischen Abdrängen der Solteilchen beim Einbringen der Glasperle ab, so kann die Ausrichtung durch verschieden starke thermische Molekularbewegung zu beiden Seiten der Phasengrenze erklärt werden. Das Teilchen erfährt von einer Seite einen stär-

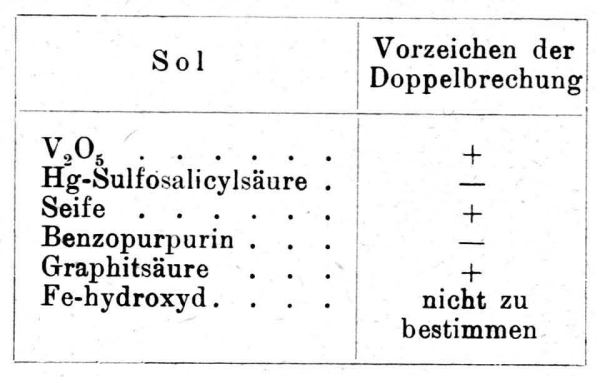

Tab. 3. Ausrichtung durch Phasengrenzen.

keren Druck, wird gegen die Glasperle gepreßt und damit zur tangentialen Einstellung gezwungen.

Außerdem können auch versehiedene Oberflächenkräfte zu beiden Seiten der Phase herrschen und die Ausrichtung bewirken. Auch elektrische Aufladungen der Wand müssen berücksichtigt werden. Versuche mit Gold an Stelle des Glases zeigten keine wahrnehmbare Doppelbrechung. Gute Effekte kann man hingegen um Luftblasen wahrnehmen. Bekannt ist die Doppelbrechung um Fremdkörper, wie Laft oder Sand, im Gelatinegel ${ }^{9}$. Diese Anisotropie wird auf Spannung in der an sich isotropen Gelatine zurückgeführt. Auch die gerichtete Kristallisation von Methylenblau, die $\mathrm{Z}$ ocher ${ }^{\mathbf{1 0}}$ auf einer nur in einer Richtung polierten Glasplatte erhielt, hat verwandte Ursachen.

Als Ergebnis unserer bisherigen Untersuchungen stellen wir fest: Oberflächenkräfte sind von Einfluß auf die Ausrichtung der Solteilchen, sie können aber eine Erklärung für die verschiedene Wirkung der Elektrolyte nicht geben.

Da sowohl die Solstäbchen wie die ausrichtenden Ionen geladene Teilchen darstellen, liegt der Gedanke nahe, die verschiedene Einstellung im Gel elektrostatisch zu deuten. Von dem sich lösenden Salz diffundieren Kationen und Anionen verschieden schnell in die Lösung, bis das dadurch entstehende Diffusionspotential (Nernst) das schnellere Ion hemmt und das langsamere beschleunigt. Beide wandern dann mit gleicher Geschwindigkeit. Die Ladung des vorauseilenden Ions müßte durch Anziehung oder Abstoßung die Orientierung der Solteilchen bedingen. Ein $\mathrm{V}_{2} \mathrm{O}_{5}$ Stäbchen besitzt an seiner Oberfläche negative Ladungen, die von einer diffusen Schicht von positiven Gegenionen kompensiert werden. Beim

${ }^{9} \mathrm{H} . \mathrm{K}$ u h n, Kolloidchem. Taschenbuch 1935, S. 231.

${ }_{10}$ H. Z o c h e r, Kolloid-Z. 36, 201 ( $\mathrm{Z}$ s i g mondyFestschrift). 
Annähern von positiven Ionen wird durch Abstoßung die Ladungswolke um das Teilchen verschoben und die negative Ladung des Stäbchens angezogen. Da die Anziehung nach dem Coulombschen Gesetz mit dem Quadrat der Entfernung abnimmt, ist sie an der den Kationen zugewandten Spitze des Kriställchens am größten. Dieses dreht sich und richtet sich dann radial

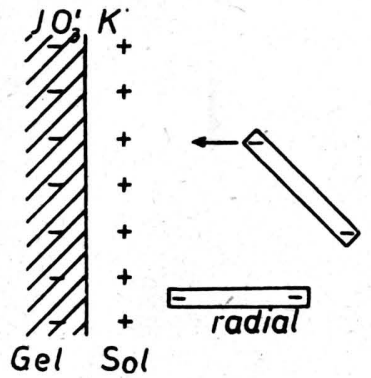

Abb. 5.

Radiale Ausrichtung durch Anziehung.

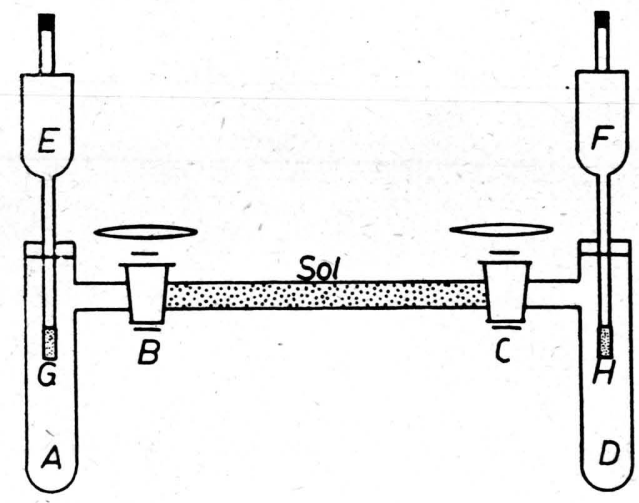

Abb. 7. Versuchsanordnung zur Messung von Diffusionspotentialen in Solen.

aus. Anschließend wird das Teilchen durch Flockung und Gelbildung festgelegt (Abb. 5).

Demgegenüber wirken Anionen, nachdem sie die positive, frei bewegliche Ladungswolke am Stäbchen neutralisiert haben, abstoßend auf das Teilchen, das sich dadurch tangential einstellt (Abb.6). Entladen und damit geflockt wird das Sol nur von seinen Gegenionen. Bei $\mathrm{KJO}_{3}$ geschieht dies von dem gleichen Ion, welches auch ausrichtet; bei $\mathrm{LiCl}$ enst von dem langsameren Kation. Dies kann aber keine nochmalige Anderung der Richtung bewirken, da die ungleiche Ladungsverteilung nur an der Diffusionsfront herrscht.
Der orientierende Einfluß geht also nur von der ersten Schicht des wegdiffundierenden Elektrolyten aus. Um diese Hypothese zu beweisen, war es erforderlich, Diffusionspotentiale in Kolloiden zu messen und festzustellen, ob ein $\mathrm{Zu}$ sammenhang mit dem Vorzeichen der Doppelbrechung besteht. Bei positiven Potentialen wandert das Kation voraus, was bei dem negativ geladenen $\mathrm{V}_{2} \mathrm{O}_{5}$ radiale Einstellung und damit negative Doppelbrechung ergeben muß. Negatives Potential wird durch schneller wandernde. Anionen erzeugt. Dieses hat tangentiale Einstellung und damit positive Doppelbrechung zur Folge. Eisenoxydsol muß entsprechend seiner positiven Ladung die umgekehrten Erscheinungen zeigen.

Die bei der Diffusion auftretenden Spannungen wurden mit der in Abb. 7 gezeigten Versuchsanordnung gemessen. Das Gefäß A war mit dem jeweils untersuchten Salz in gesättigter Lösung gefüllt, dessen Diffusionspotential gemessen werden sollte. Nach dem Öffnen der Hähne konnte der Elektrolyt in das Sol diffundieren, das sich zwischen B und C befand. Das Glas D enthielt destilliertes Wasser, in dem sich im Laufe des Versuches soviel Elektrolyt anreicherte, daß seine Leitfähigkeit für die Messung ausreichte. Die ganze Anordnung wurde über zwei Tonstifte G und $\mathrm{H}$ mit den gesättigten Kalomelelektroden $\mathrm{E}$ und $\mathrm{F}$ leitend verbunden. Potentialsprünge treten auf bei $\mathrm{G}, \mathrm{B}, \mathrm{C}$ und $\mathrm{H}$. Die bei $\mathrm{C}$ und $\mathrm{H}$ bleiben konstant und beeinflussen das Meßergebnis stets gleichmäßig und im selben Sinne. Die Spannungsdifferenz bei $B$ soll gemessen werden, die bei $G$ geht als Fehler in das Ergebnis ein.

Die auftretenden Potentialdifferenzen wurden mit einem Ionometer gemessen. Das Ergebnis der Messungen ist in Tab. 4 für $\mathrm{V}_{2} \mathrm{O}_{5}$ und in Tab.5 für Eisenoxydsol für eine Reihe von Salzen eingetragen. Zu Beginn der Messung ändert sich die Spannung noch merklich und wird erst nach 60 Min. konstant, wenn Anzahl und Geschwindigkeit der wegwandernden Ionen sich nicht mehr ändern. Da in einer flachen Glasschale andere Verhältnisse vorliegen als in dem Glasrohr BC, bleibt dahingestellt, ob diese anfängliche Potentialänderung bei unseren Versuchen theoretisches Interesse besitzt. Immerhin könnte sie eine Erklärung für die häufig beobachtete Änderung der Doppelbrechung mit wachsendem Gel geben. Versuche, das Diffusionspotential direkt in der Schale zu messen, lieferten unbrauchbare Werte. Es bildet 


\begin{tabular}{|c|c|c|}
\hline $\mathrm{mV}$ & Ionenpaar & $\begin{array}{l}\text { Vorzeichen der } \\
\text { Doppelbrechung }\end{array}$ \\
\hline+68 & $\mathrm{KH}_{2} \mathrm{PO}_{4}$. & - \\
\hline+49 & $\mathrm{~K}_{2} \mathrm{Cr}_{2} \mathrm{O}_{7}$ & - \\
\hline+47 & $\mathrm{~K}_{3}^{2} \mathrm{Fe}(\mathrm{CN})_{6}$ & - \\
\hline+45 & $\mathrm{~K}_{4} \mathrm{Fe}(\mathrm{CN})_{6}$ & - \\
\hline+43 & $\mathrm{~K}\left(\mathrm{CH}_{3} \mathrm{COO}\right)$ & - \\
\hline+26 & $\mathrm{KJO}_{3} \cdot$ & - \\
\hline+23 & $\mathrm{MgSO}_{4}$ & + \\
\hline+17 & $\mathrm{~K}_{2} \mathrm{~S}_{2} \mathrm{O}_{8}$ & - \\
\hline+14 & $\mathrm{KNO}_{3} \cdot \cdot$ & + \\
\hline+12 & $\mathrm{KCl} \cdot \cdot$ & + \\
\hline+7 & $\mathrm{Tl}_{2} \mathrm{SO}_{4} \cdot$ & - \\
\hline+4 & $\mathrm{Th}\left(\mathrm{NO}_{3}\right)_{4}$ & - \\
\hline-14 & $\mathrm{Ca}\left(\mathrm{NO}_{3}\right)_{2}$ & + \\
\hline-39 & $\mathrm{LiCl} \cdot \cdot$ & + \\
\hline $\begin{array}{l}-41 \\
-73\end{array}$ & $\mathrm{MnCI}_{2}$ & + \\
\hline-75 & $\mathrm{Sr}(\mathrm{OH})_{2}$ & + \\
\hline
\end{tabular}

Tab. 4. Gemessene Diffusionspotentiale und Doppelbrechung in $\mathrm{V}_{2} \mathrm{O}_{5}-\mathrm{Sol}(0,1 \%)$.

sich um den Tonstift durch das KCl der Elektrode ein Gel, das unkontrollierbare Membranpotentiale erzeugt. Deshalb wurde in das Gefäß D destilliertes Wasser gegeben, um eine direkte Verbindung Elektrode/Sol zu vermeiden.

In den Tab. 4 und 5 ist in befriedigendem Maße experimentell bestätigt, was die Theorie fordert. Die negativ doppelbrechendén Salze liegen beim $\mathrm{V}_{2} \mathrm{O}_{5}$ mehr zur positiven Seite des Diffusionspotentials hin. Die positiv doppelbrechenden häufen sich vor allem im Gebiete negativer Spannung an. Wie erwartet, zeigt Eisenoxydsol gerade die umgekehrten Verhältnisse, denn es ist entgegengesetzt geladen. Mehr konnte von diesen Messungen nicht erwartet werden. Die Fehlergrenze wird mit $\pm 5-10$ Millivolt angenommen, da die Potentialsprünge bei $\mathrm{G}, \mathrm{C}$ und $\mathrm{H}$ in das Ergebnis mit eingehen. Innerhalb dieser Fehlergrenzen liegt auch die Reproduzierbarkeit der Meßergebnisse für unsere Anordnung. Durch diese Ungenauigkeit läßt sich schwer etwas dar- über sagen, ob und wieweit ein Unterschied zwischen den beiden Solen für ein und denselben Elektrolyten besteht.

Wenn bei den Messungen von gesättigten Salzlösungen ausgegangen wurde, so sollte damit eine möglichst große Übereinstimmung mit den Versuchen in flachen Schalen erzielt werden.

Sollte unsere Behauptung richtig sein, daß das schneller wandernde Ion durch elektrostatische Kräfte Ausrichtung der Solteilchen bedingt, so

\begin{tabular}{|c|c|c|}
\hline $\mathrm{mV}$ & Ionenpaar & $\begin{array}{l}\text { Vorzeichen } \\
\text { der Doppel- } \\
\text { brechung }\end{array}$ \\
\hline+80 & $\mathrm{Ce}\left(\mathrm{SO}_{4}\right)_{2}$ & + \\
\hline $\begin{array}{l}+47 \\
+4\end{array}$ & $\mathrm{Na}_{3}$-Citrat & + \\
\hline+46 & $\mathrm{~K}_{2} \mathrm{SO}_{4}$ & + \\
\hline+43 & $\mathrm{~K}_{2}^{2} \mathrm{Cr}_{2} \mathrm{O}_{\tau}$ & + \\
\hline+42 & $\mathrm{~K}_{3}^{-} \mathrm{Fe}(\mathrm{CN})_{6}$ & - \\
\hline $\begin{array}{l}+38 \\
+33\end{array}$ & $\mathrm{~K}_{4} \mathrm{Fe}(\mathrm{CN})_{6} \cdot$ & + \\
\hline $\begin{array}{l}+35 \\
+30\end{array}$ & $\begin{array}{l}\mathbf{h}_{2} \mathrm{~S}_{2} \mathrm{U}_{8} \\
\mathbf{K}\left(\mathrm{CH}_{3} \mathrm{COO}\right)\end{array}$ & $\bar{t}$ \\
\hline+26 & $\mathrm{Tl}_{2} \mathrm{SO}_{4}^{3}$ & + \\
\hline+23 & $\mathrm{KJ}_{3} \cdot$ & + \\
\hline+18 & $\mathrm{MoSO}_{4} \cdot \cdot$ & $\begin{array}{l}t \\
+\end{array}$ \\
\hline+14 & $\mathrm{KNO}_{3}{ }^{4}$ & - \\
\hline+13 & $\mathrm{KCl} \cdot$. & - \\
\hline+3 & $\mathrm{Ca}\left(\mathrm{NO}_{3}\right)_{2}$ & - \\
\hline-5 & $\mathrm{MnCl}_{2}$ & - \\
\hline-20 & $\mathrm{LiCl}$ & - \\
\hline $\begin{array}{l}-42 \\
-60\end{array}$ & $\underset{\mathrm{Ar}\left(\mathrm{OH} \mathrm{H}_{3}\right.}{\mathrm{Al}^{\prime}} \cdot$ & E \\
\hline & & \\
\hline
\end{tabular}

Tab.5. Gemessene Diffusionspotentiale und Doppelbrechung in Fe-Oxydsol $(1,3 \%)$.

mußte man das Vorzeichen der Doppelbrechung voraussagen können. Voraussetzung war ein möglichst großer Unterschied in der Wanderungsgeschwindigkeit von Anion und Kation. Dies traf für eine ganze Reihe von Salzen zu. Unbefriedigend war das Ergebnis aber für Ionenpaare wie $\mathrm{Na}_{2} \mathrm{CO}_{3}, \mathrm{KHSO}_{4}$ und $\mathrm{K}_{2} \mathrm{HPO}_{4}$, die isotrope Gele lieferten, obgleich ein großer Unterschied in der Beweglichkeit von Kation und Anion bestand. Sie fielen auch keineswegs in das Gebiet geringer Unterschiede der Ionenleitfähigkeiten, in welchen sich negative und positive Doppelbrechung überlagern. Diese Tatsache machte eine neue Prüfung erforderlich. Diese zeigte bei einem großen Teil der Salze, wie $\mathrm{KH}_{2} \mathrm{PO}_{4}, \mathrm{KHSO}_{4}, \mathrm{~K}\left(\mathrm{CH}_{3} \mathrm{COO}\right), \mathrm{Na}_{3} \mathrm{PO}_{4}$ in $\mathrm{V}_{2} \mathrm{O}_{5}$ Sol das theoretisch zu erwartende Ergebnis.

Man muß allerdings nur winzige Kristallsplitter verwenden und die entstandene flache Gelscheibe sofort beobachten. Diese darf nur einen kleinen Durchmesser haben. Schnell setzt sonst durch das Salz sekundär eine chemische Reaktion ein. Die Salze reagieren nicht neutral und bilden im alkalischen Gebiet Vanadate, die das entstandene Gel in Farbe und Struktur so verändern. daß es seine Doppelbrechung verliert. Aus diesem Grunde werden auch andere, später isotrope Gele, wie durch $\mathrm{Na}_{2} \mathrm{CO}_{3}$ oder KCN in Vanadinpentoxydsol gebildete, anfangs doppelbrechend sein. Hier- 


\begin{tabular}{|c|c|c|}
\hline $\mathrm{mV}$ & Ionenpaar & $\begin{array}{l}\text { Vorzeichen } \\
\text { der Doppel- } \\
\text { brechung }\end{array}$ \\
\hline $\begin{array}{l}+20 \\
+16 \\
+15 \\
+12 \\
+10 \\
+8 \\
+6 \\
+4 \\
+2\end{array}$ & 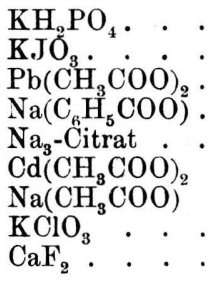 & $\begin{array}{l}\overline{-} \\
\overline{-} \\
\overline{-} \\
\overline{+} \\
\overline{+}\end{array}$ \\
\hline-2 & $\mathrm{Tl}_{2} \mathrm{SO}_{4} \cdot$ & - \\
\hline $\begin{array}{l}-4 \\
-5\end{array}$ & $\left(\mathrm{NH}_{4}\right)_{2} \mathrm{~S}_{2} \mathrm{O}_{8} \cdot$ & \pm \\
\hline-6 & $\mathrm{Sr}\left(\mathrm{NO}_{3}\right)_{2}$ & $\bar{t}$ \\
\hline-7 & $\mathrm{~K}_{2} \mathrm{~S}_{2} \mathrm{O}_{8} \cdot \cdot \cdot \cdot$ & - \\
\hline $\begin{array}{l}-8 \\
-11\end{array}$ & $\mathrm{~K}_{3} \mathrm{Fe}(\mathrm{CN})_{6} \cdot \cdot$ & $\bar{t}$ \\
\hline $\begin{array}{l}-11 \\
-12\end{array}$ & $\begin{array}{ll}\mathrm{NaNO}_{3} & \cdot \\
\mathrm{CdSO}_{4} & \vdots\end{array}$ & $\begin{array}{l}+ \\
+\end{array}$ \\
\hline-13 & $\mathrm{NaCl}$ & + \\
\hline-14 & $\mathrm{~K}_{4} \mathrm{Fe}(\mathrm{CN})_{6} \ldots$ & -. \\
\hline $\begin{array}{l}-15 \\
-19\end{array}$ & $\mathrm{BaCl}_{2} \cdot \cdot \cdot$ & + \\
\hline $\begin{array}{l}-19 \\
-25\end{array}$ & $\begin{array}{ll}\mathrm{LiCl} \\
\mathrm{Th}\left(\mathrm{NO}_{3}\right)_{4}\end{array}$ & \pm \\
\hline-30 & $\mathrm{Sr}(\mathrm{OH})_{2} \cdot$ & + \\
\hline
\end{tabular}

Tab. 6. Berechnete Diffusionspotentiale und beobachtete Doppelbrechung.

mit waren diese Widersprüche gedeutet. Die betreffenden Salze verändern chemisch und optisch die Solteilchen.

Da die Messung des Diffusionspotentials mit Fehlern behaftet war, schien es angebracht, eine Kontrolle durch berechnete Werte zu schaffen. Das Diffusionspotential hängt von folgenden Größen ab:

$$
\pi=\frac{u-v}{u+v} \cdot \frac{R T}{F} \cdot \ln \frac{c}{c^{\prime}} .
$$

$u, v=$ Wanderungsgeschwindigkeit der Ionen,

$R=$ Gaskonstante, $T=$ abs. Temp.,

$F=$ Faraday-Konstante,

$c, c^{\prime}=$ Konzentration,

Es kommt bei unseren Versuchen nur auf relative Werte an, deshalb wird $\ln c / c^{\prime}=1$ gesetzt, und man erhält die Gleichung

$$
\pi=\frac{u-v}{u+v} \cdot 0,058 \text { für } t=20^{\circ} \mathrm{C} .
$$

Die hiernach berechneten Diffusionspotentiale sowie die durch die zugehörigen Salze erzeugte Doppelbrechung zeigt Tab. 6. Die zugrunde gelegten Wanderungsgeschwindigkeiten $u$ und $v$ wurden den Tab. von Londolt-Börnstein entnommen.
Auf den ersten Blick scheint zwischen den berechneten Werten und der Doppelbrechung kein System zu liegen. Ordnet man die Salze jedoch nach ihrer Ionenwertigkeit wie in Tab. 7 und 8. so findet man eine Beziehung zwischen Wanderungsgeschwindigkeit und Anisotropie. Allerdings liegt der Umkehrpunkt des Vorzeichens der Doppelbrechung bei den verschiedenen Wertigkeiten verschieden hoch. Die experimentell gefundenen Kurven zeigen diesen Unterschied nicht so ausgeprägt. Besonders die mehrwertigen Anionen des $\mathrm{K}_{3} \mathrm{Fe}(\mathrm{CN})_{6}, \mathrm{~K}_{4} \mathrm{Fe}(\mathrm{CN})_{6}$ und $\mathrm{K}_{2} \mathrm{~S}_{2} \mathrm{O}_{8}$ werden durch das Sol so stark gehemmt, daß, nach dem ionotropen Effekt zu urteilen, das Kation vorauseilt.

Sieht man das entstandene Gel als Membran mit variierter Durchlässigkeit für Ionen an, so lassen sich die Erscheinungen auch von der Theorie des Membranpotentials verstehen. Aufladung der Membran veranlaßt die Ausrichtung der Solteilchen. Dies bringt aber keinen prinzipiellen neuen Gesichtspunkt zur Erklärung der Ionotropie, denn in der Gleichung

$$
\pi=\frac{u-v}{u+v} \cdot R T \ln \frac{c_{1}}{c_{2}}
$$

tritt lediglich für $u$ und $v$ die Wanderungsgeschwindigkeit der Ionen durch die Membran. Ist die Wanderungsgeschwindigkeit im Sol anders als im Wasser, so erhält man eine Erklärung für die Diskrepanz zwischen berechnetem und gefundenem Potential bei den oben genannten Ionenpaaren. Im Gleichgewichtszustand gehen die Membranpotentiale in Donnan-Potentiale über, wenn mindestens eine Ionenart an der freien Beweglichkeit gehindert wird. Das träfe bei den noch teilweise geladenen Kolloidionen im Gel zu. Der Einfluß von Donnan-Gleichgewichten wurde nicht untersucht, da ihre Einstellung längere Zeit braucht. Ionotrope Gele bilden sich aber in kurzer Zeit.

In $\mathrm{Fe}(\mathrm{OH})_{3}$-Sol zeigen einige Salze wie $\mathrm{CdSO}_{4}$. $\mathrm{K}_{2} \mathrm{SO}_{4}, \mathrm{KNO}_{3}$ mit wachsendem Gel eine Umkehrung der Doppelbrechung. Innen ist das Gel positiv und außen negativ doppelbrechend oder umgekehrt. Dies wird auf eine Anderung der Wanderungsgeschwindigkeit ihrer Ionen zurückzuführen sein. Chemische Reaktionen oder Adsorption hemmen das ursprünglich vorauseilende Ion. 


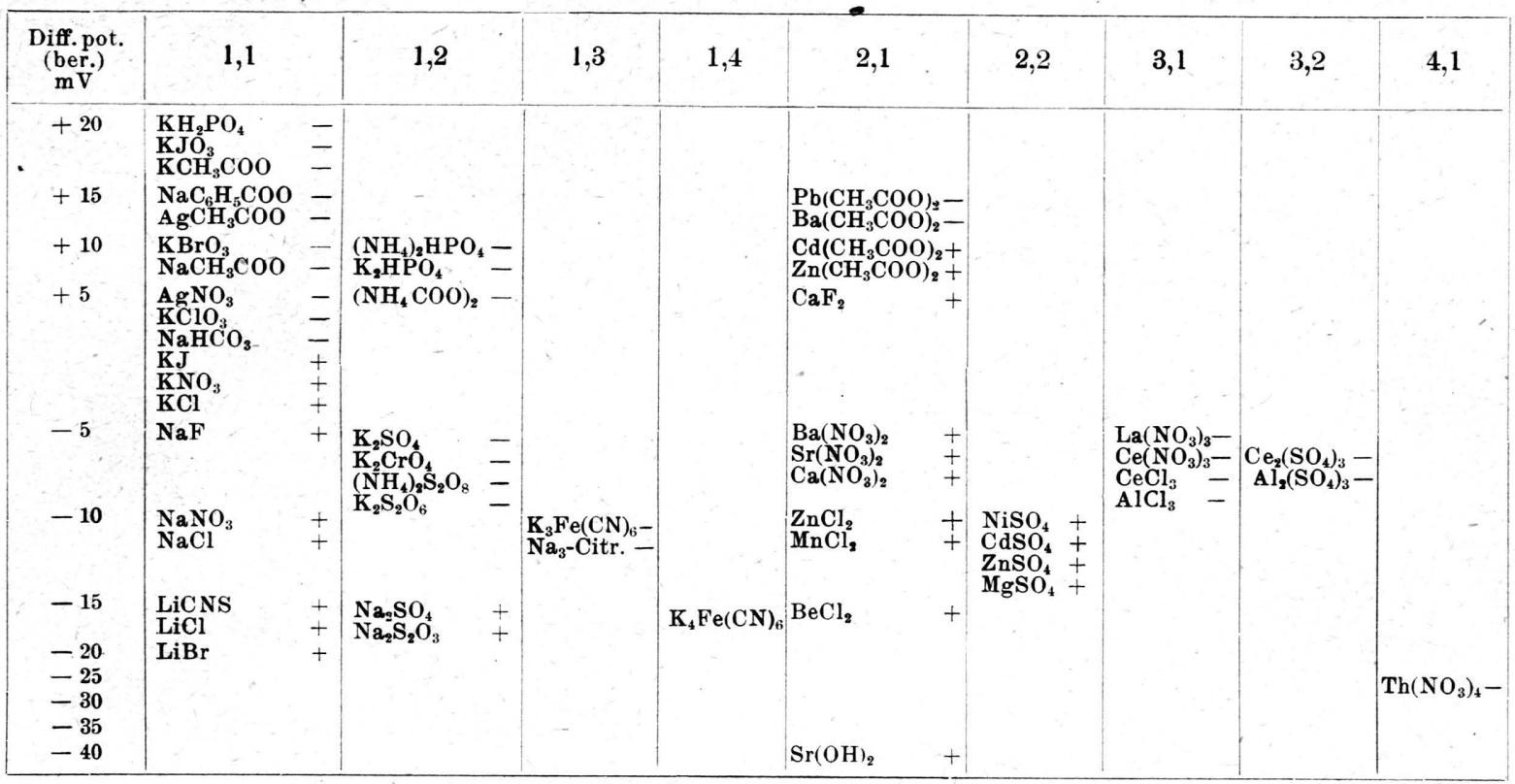

Tab. 7. Berechnete Diffusionspotentiale und Doppelbrechung in $\mathrm{V}_{2} \mathrm{O}_{5}$, aufgeteilt in Ionenwertigkeit.

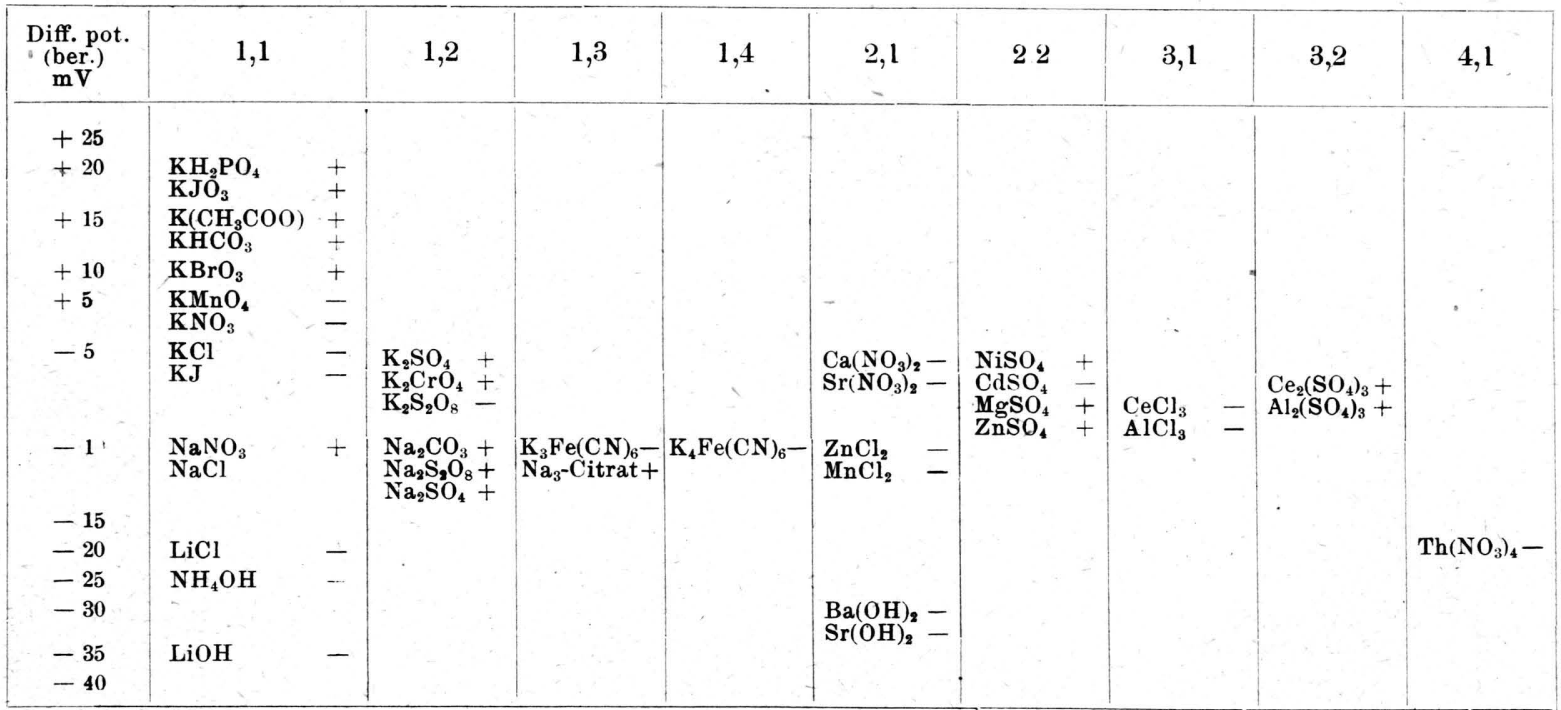

Tab. 8. Berechnete Diffusionspotentiale und Doppelbreshung in Eisenoxydsol (1,3\%), aufgeteilt in Ionenwertigkeit.

Zweifellos sprechen bei der Ausrichtung auch andere Faktoren, wie Ladung, Hydratation und Polarisierbarkeit der richtenden Ionen mit.

Aus der Reihe der gemessenen Potentiale der Tab. 7 u. 8 sind zur besseren Übersicht zwei charakteristische Beispiele herausgegriffen (S. 402 oben).

Für Eisenoxydsol mit positiver Ladung der Teilchen ist Ausrichtung und Doppelbrechung für $\mathrm{KJO}_{3}$ und $\mathrm{LiCl}$ der von $\mathrm{V}_{2} \mathrm{O}_{5}$ entgegengesetzt.
Trifft das bei anderen Salzen nicht immer zu, so ist entweder die Aquivalentleitfähigkeit der beiden Ionen des zugesetzten Salzes nahezu gleich groß, oder die Wanderungsgeschwindigkeit im Sol weicht von der im Wasser ab. Die in den Tabellen vorkommenden Abweichungen führten zu Untersuchungen über den Einfluß des elektrokinetischen Potentials. Endgültige Ergebnisse liegen noch nicht vor. 


\begin{tabular}{|c|c|c|c|c|c|}
\hline Ionenpaare & $\begin{array}{l}\text { Äquivalentleitf. } \\
\text { in } \mathrm{H}_{2} \mathrm{O}\end{array}$ & $\begin{array}{l}\text { Größere Wanderungs- } \\
\text { geschwindigkeit. }\end{array}$ & $\begin{array}{l}\text { Elektrostat. } \\
\text { Einwirkung }\end{array}$ & $\begin{array}{l}\text { Teilchen- } \\
\text { Einstellung }\end{array}$ & $\begin{array}{l}\text { Vorzeichen der } \\
\text { Doppelbrechg. }\end{array}$ \\
\hline $\mathrm{KJO}_{3} \cdot \cdot \cdot$ & $\begin{array}{l}\mathrm{K}^{\prime}=73,5 \\
\mathrm{JO}_{3}=41,0\end{array}$ & $\begin{array}{l}\text { K wandert schneller, } \\
\text { Aufladung des Gel- } \\
\text { randes positiv }\end{array}$ & $\begin{array}{l}\text { Gel zugewandte } \\
\text { Spitze d. Teil- } \\
\text { chen stärker } \\
\text { angezogen }\end{array}$ & radial & - \\
\hline $\mathrm{LiCl}$. & $\begin{array}{l}\mathrm{Li}^{\prime}=38,5 \\
\mathrm{Cl}=76,3\end{array}$ & $\begin{array}{l}\mathrm{Cl} \text { wandert schneller, } \\
\text { Gelrand negativ }\end{array}$ & $\begin{array}{l}\text { Gel zugewandte } \\
\text { Spitze d. Teil- } \\
\text { chen stärker } \\
\text { abgestoßen }\end{array}$ & tangential & + \\
\hline
\end{tabular}

Zusammenfassend stellen wir fest, daß die Ausrichtung der kolloiden Stäbchen durch eine elektrostatische Anziehung oder Abstoßung der schneller wandernden Ionen oder durch eine Aufladung des Gelrandes durch Membranpotentiale bedingt wird. In der eingenommenen Lage wird dann das Kolloidteilchen durch Koagulation fixiert. $\mathrm{Ob}$ man eine ungleichmäßige Verteilung der Ladung an der Oberfläche des Solteilchens oder Multipolmomente annimmt, die ebenfalls eine verschiedene Orientierung im inhomogenen Feld erfahren, bleibt für den Endeffekt gleichgültig. Dipole sind ausgeschlossen, weil sie sich nur in Richtung des Feldes, also radial, einstellen würden.

Nach diesen Erfahrungen müssen sich alle anisodiametrischen Teilchen durch Elektrolyte richten lassen, wenn sie nur eine Ladung tragen. Bei isotropen anisodiametrischen Solen, die keine Doppelbrechung zeigen, müßte dies noch durch Röntgenstrukturbestimmung bestätigt werden.

Einige Röntgendiagramme von ionotropen $\mathrm{V}_{2} \mathrm{O}_{5}$ Gelen ergaben verwaschene Ringe, die sich dem $\mathrm{V}_{2} \mathrm{O}_{5}$ - Gitter zuordnen lassen. Die Debye- Scherrer-Ringe haben Verdickungen, welche eine Ausrichtung der Kolloidteilchen bestätigen.

Obgleich bei $\mathrm{V}_{2} \mathrm{O}_{5}$-Solen sehr hohe Dielektrizitätskonstanten gemessen wurden ${ }^{11}$, erscheinen die dielektrischen Eigenschaften des Sols von untergeordneter Bedeutung bei der Ausrichtung

${ }^{11}$ Er r e r a, Kolloid-Z. 31, 59 [1922]. der Teilchen zu sein, sonst müßte die Orientierung stets in die Stellung führen, bei welcher die Richtung größter Elektrizitätskonstanten $\varepsilon$ und elektrisches Feld übereinstimmen.

Diese Lage fällt nach der Maxwellschen Beziehung:

$$
n=\sqrt{\varepsilon \cdot 11}
$$

mit der längeren Indexachse des Kriställchens zusammen, sofern die Permeabilität von untergeordneter Bedeutung ist. Das bedeutet, daß längere Indexachse und elektrisches Feld parallel liegen. Daraus resultiert für $\mathrm{V}_{2} \mathrm{O}_{5}$ und $\mathrm{Fe}(\mathrm{OH})_{3}$ radiale Einstellung, für $\mathrm{Hg}$-Sulfosalicylsäure und Benzopurpurin tangentiale Anordnung.

Diese Beobachtungen werden tatsächlich im elektrischen Feld eines Kondensators gemacht ${ }^{12}$.

In ionotropen Gelen findet man dies aber nicht immer, woraus in diesem Falle auf den geringen Einfluß der Dielektrizitätskonstanten zu schließen ist.

Die verschiedene Ausrichtung der Solteilchen in Abhängigkeit von dem Diffusionspotential der Elektrolyte wurde bisher nur bei Vanadinpentoxyd und Eisenoxyd beobachtet.' Die anderen untersuchten Sole, wie Quecksilbersulfosalicylsäure, Graphitsäure und Benzopurpurin, ergaben isotrope Gele oder solche mit tangential angeordneten Teilchen.

12 F ü r th u. B l üh, Kolloid-Z. 32, 337 [1923]. 